\title{
Role of the Alkali Metal Cation in the Early Stages Crystallization of Halide Perovskites
}

\section{- Supporting Information -}

Marion A. Flatken ${ }^{\mathrm{a}}$, Eros Radicchi ${ }^{\mathrm{b}}$, Robert Wendt ${ }^{\mathrm{a}}$, Ana Guilherme Buzanich ${ }^{\mathrm{c}}$, Eneli Härk ${ }^{\mathrm{a}}$, Jorge Pascual $^{\mathrm{d}}$, Florian Mathies ${ }^{\mathrm{a}}$, Oleksandra Shargaieva ${ }^{\mathrm{a}}$, Albert Prause ${ }^{\mathrm{e}}$, André Dallmann ${ }^{\mathrm{f}}$, Filippo De Angelis ${ }^{\mathrm{g}}$, Armin Hoella, Antonio Abate ${ }^{\mathrm{a} *}$

a M. Flatken, Dr. R. Wendt, Dr. E. Härk, Dr. F. Mathies, Dr. O. Shargaieva, Dr. A. Hoell, Prof. A. Abate Helmholtz-Zentrum Berlin für Materialien und Energie GmbH

Hahn-Meitner-Platz 1, 14109 Berlin, Germany

E-mail: antonio.abate@,helmholtz-berlin.de

b Dr. E. Radicchi

Computational Laboratory for Hybrid/Organic Photovoltaics (CLHYO), Istituto CNR di Scienze e Tecnologie Chimiche "Giulio Natta" (CNR-SCITEC),

Via Elce di Sotto 8, 06123 Perugia, Italy

c Dr. A. Guilherme Buzanich

Bundesanstalt für Materialforschung und - prüfung (BAM)

12489, Berlin, Germany

d Dr. J. Pascual,

Institute for Chemical Research, Kyoto University, Gokasho, Uji, Kyoto 611-0011, Japan

e A. Prause, Technische Universität Berlin Straße des 17. Juni 135, 10623 Berlin

f Dr. A. Dallmann Institut für Chemie Humboldt Universität zu Berlin Brook-Taylor-Str. 2, 12489 Berlin, Germany

g Dr. F. De Angelis, Department of Chemistry, Biology and Biotechnology, University of Perugia, Via Elce di Sotto 8, 06123 Perugia, Italy;

CompuNet, Istituto Italiano di Tecnologia, Via Morego 30, 16163 Genova, Italy;

Department of Mechanical Engineering, College of Engineering, Prince Mohammad Bin Fahd University, P.O. Box 1664 Al Khobar 31952, Kingdom of Saudi Arabia. 


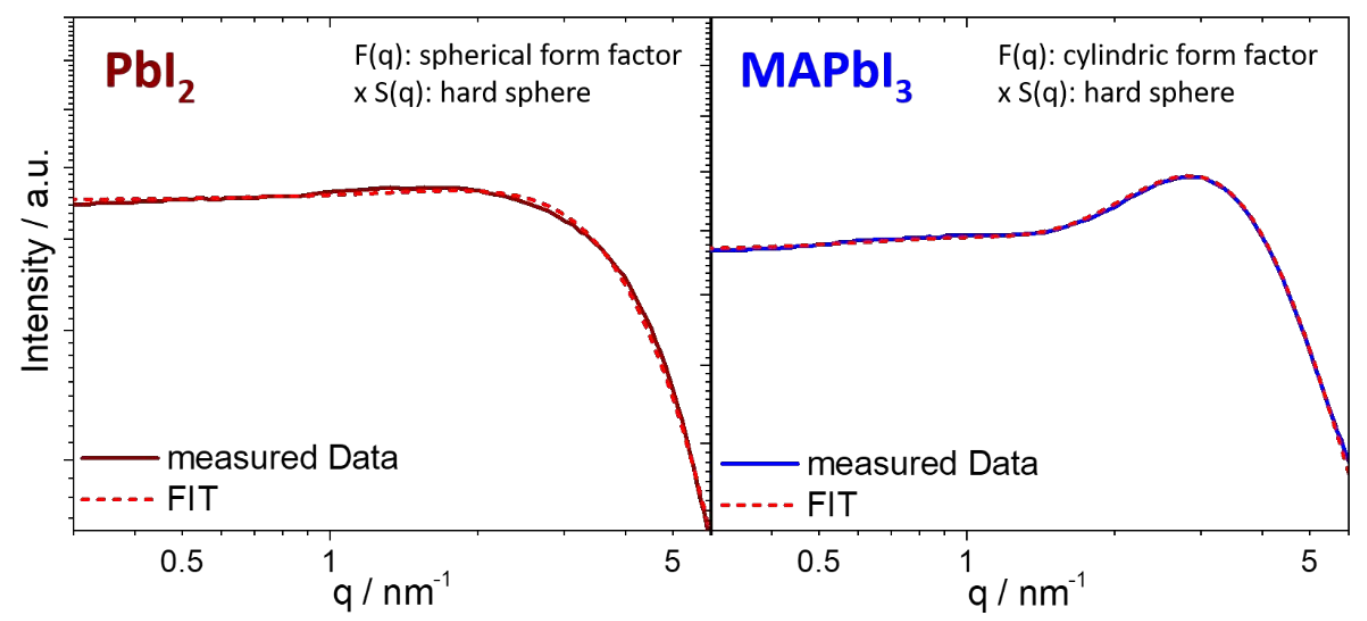

\begin{tabular}{l|ccc|cc|c} 
& \multicolumn{3}{|c|}{$\mathrm{F}(\mathrm{q})$ Parameters } & \multicolumn{2}{c|}{$\mathrm{S}(\mathrm{q})$ Parameters } & Variance FIT \\
\hline sample & $\mathbf{s}$ & $\mathbf{R} / \mathbf{n m}$ & $\mathbf{L} / \mathbf{n m}$ & $\mathbf{R}_{\mathrm{HS}} / \mathbf{n m}$ & $\mathbf{F}_{\mathbf{p}}$ & $\mathbf{\sigma}^{\mathbf{2}}$ \\
\hline $\mathbf{P b l}_{2}$ & 0.022 & 0.407 & n.a. & 0.68 & 0.030 & $4.4 \times 10^{-9}$ \\
\hline MAPbl $_{3}$ & 0.012 & 0.145 & 0.64 & 0.77 & 0.037 & $1.7 \times 10^{-10}$ \\
\hline
\end{tabular}

Figure S1. Model fit of neutron scattering curves of $\mathrm{PbI}_{2}$ and $\mathrm{MAPbI}_{3}$ precursors. Model fits were performed using SASfit software ${ }^{1}$. The fit parameters for each sample are provided below. For the form factor the width parameter s, the radius $\mathrm{R}$ and the length of the cylindrical form factor $\mathrm{L}$ are tabulated and for the structure factor the hard-sphere interaction radius $\mathrm{R}_{\mathrm{HS}}$ and the volume fraction $f_{p}$ as well as the overall variance of the fit $\sigma^{2}$.

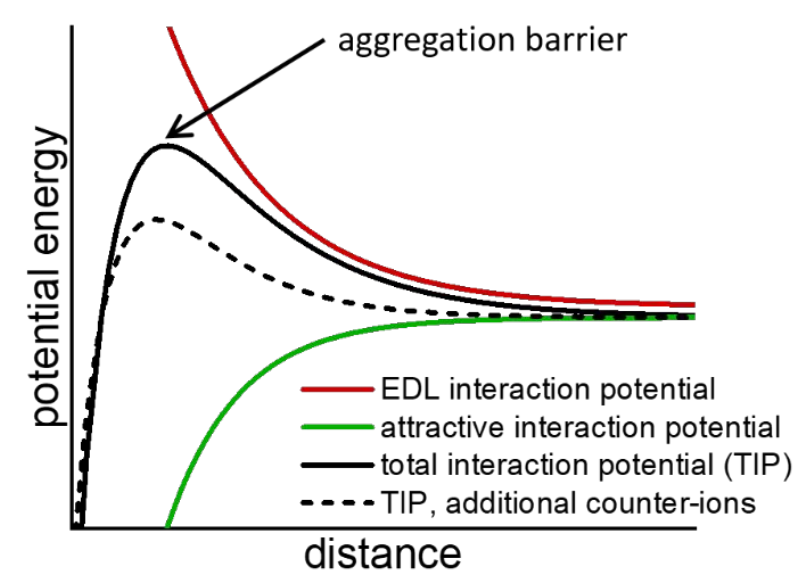

Figure S2. Total interaction potential (TIP) as sum of the attraction potential and the repulsion potential (EDL). Change of the TIP as a consequence of counter-ion addition is presented by the dashed black line.

The TIP depicts the variation of colloidal interaction energy with respect to the distance between the colloid particles. According to the DLVO theory, TIP comprises the interplay of Van der Waals attraction and electrostatic repulsion (in the Figure S2 described as EDL interaction potential) between colloid particles. The electrostatic repulsion, which is caused by the overlap of the electrochemical diffuse layers surrounding the charged NP can be influenced, for instance, through the addition of counter-ions. In fact, the addition of counter-ions to a colloidal system weakens the electrostatic repulsion and, thus, reduces the maximum energy of the TIP, the aggregation barrier (depicted as a black, dotted line in Figure S2). 


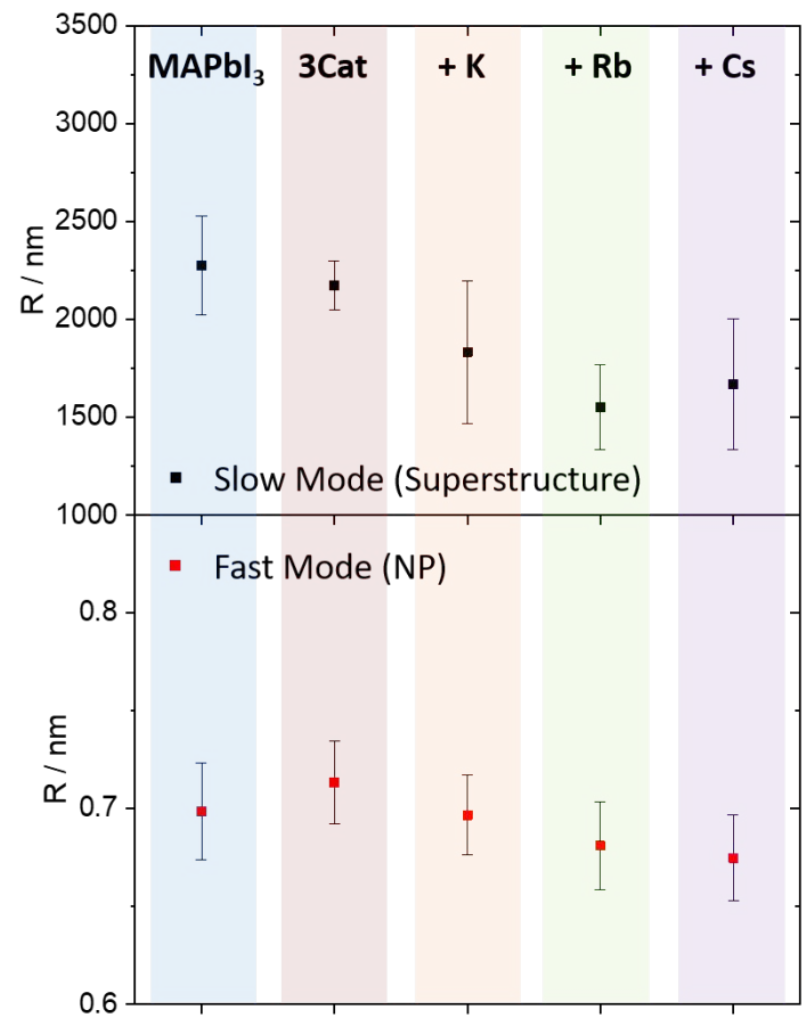

Figure S3. Average sizes determined by DLS. 

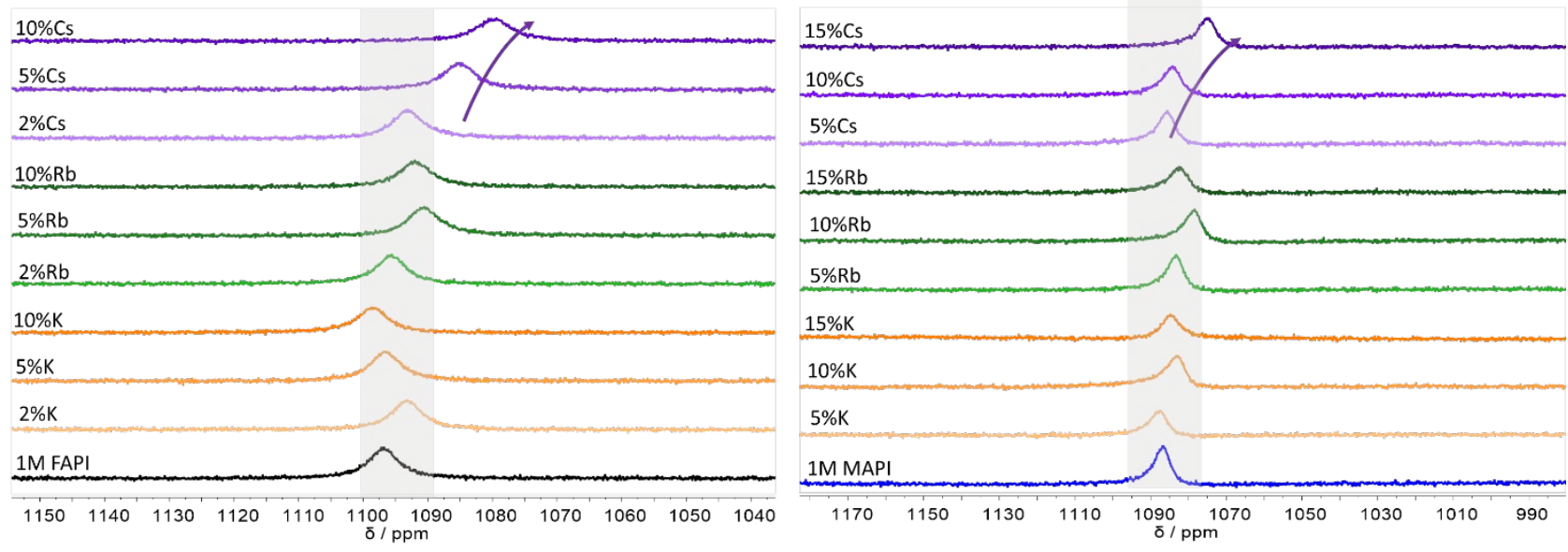

Figure S4. ${ }^{207} \mathrm{~Pb}-\mathrm{NMR}$ on precursor solutions. $\mathrm{FAPbI}_{3}$ and $\mathrm{MAPbI}_{3}$ solution with the addition of different percentages of alkali metal addition

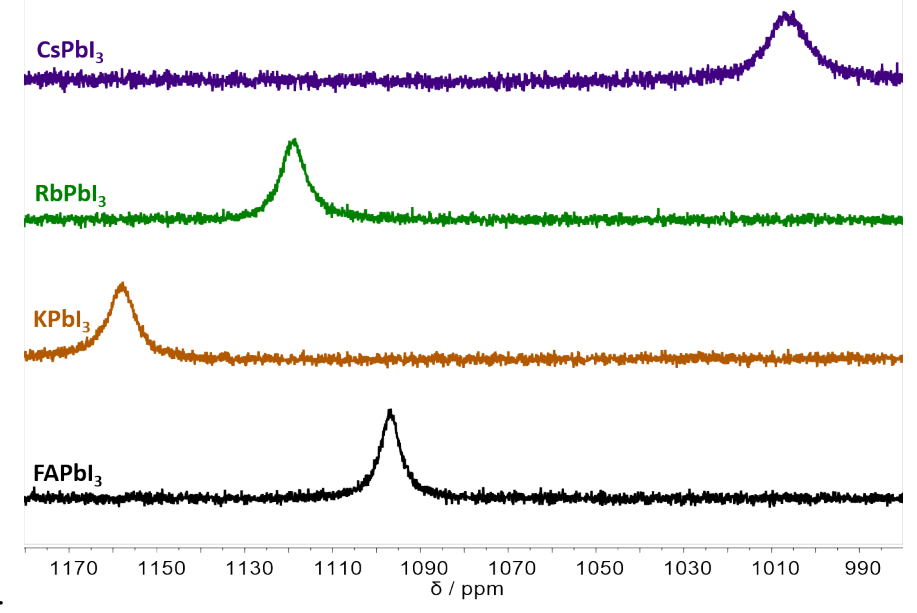

Figure S5. ${ }^{207} \mathrm{~Pb}$ NMR on Precursor solutions. $\mathrm{FAPbI}_{3}$ in comparison to complete substitution of the A-site cation by an alkali metal. 
a)

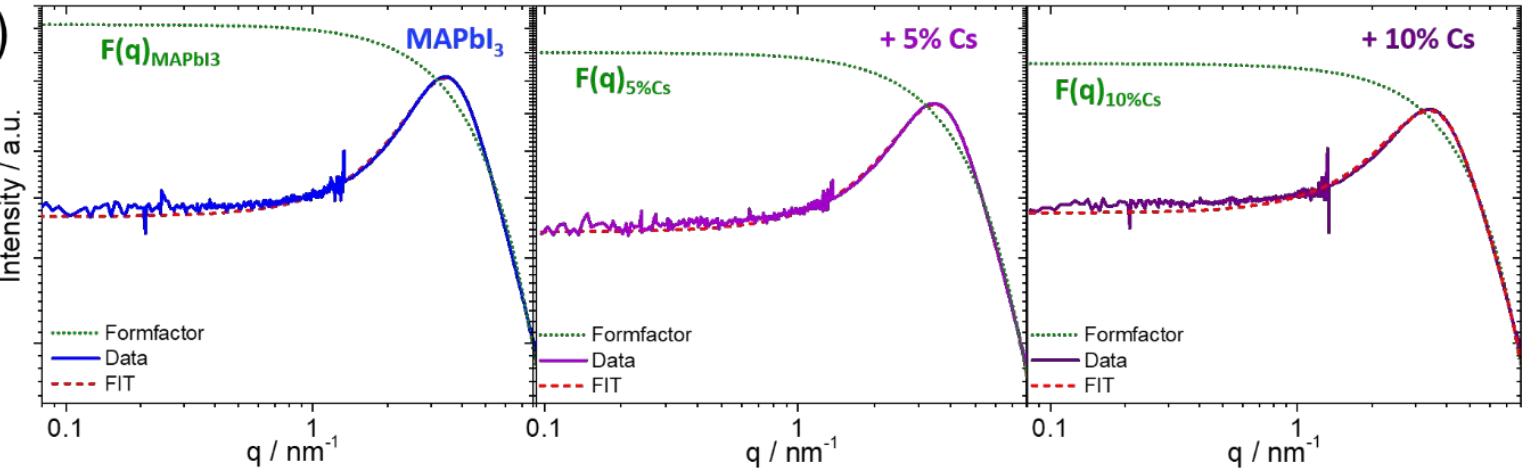

b)

c)

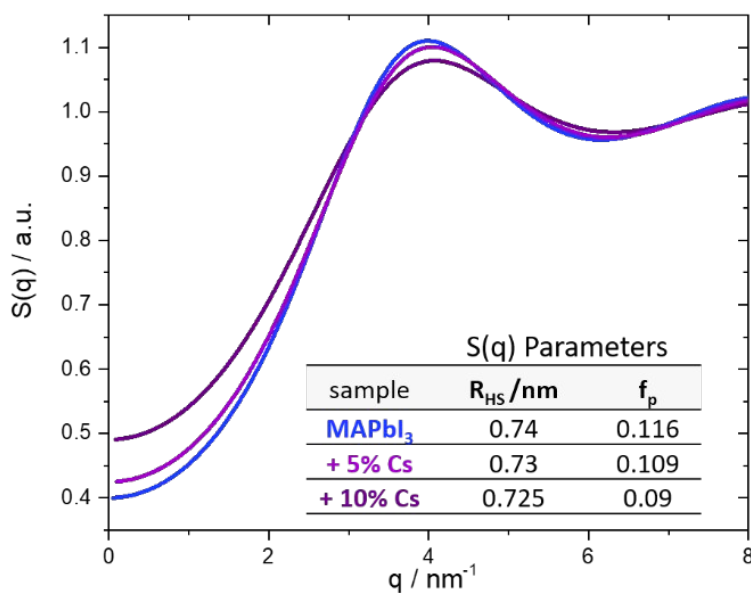

Cylindrical

Formfactor, $\mathrm{F}(\mathrm{q})$

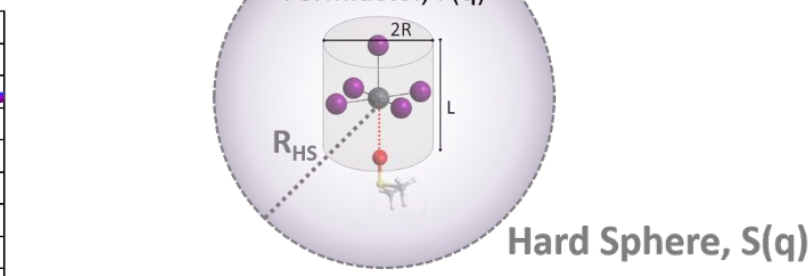

\begin{tabular}{c|ccc|c} 
& \multicolumn{3}{|c|}{$\mathrm{F}(\mathrm{q})$ Parameters } & Variance FIT \\
\hline sample & $\mathbf{s}$ & $\mathbf{R} / \mathbf{n m}$ & $\mathbf{L} / \mathbf{n m}$ & $\boldsymbol{\sigma}^{\mathbf{2}}$ \\
\hline $\mathrm{MAPbI}_{3}$ & 0.02 & 0.247 & 0.745 & $2.4 \times 10^{-12}$ \\
\hline$+5 \% \mathrm{Cs}$ & 0.01 & 0.242 & 0.720 & $1.9 \times 10^{-12}$ \\
\hline$+10 \% \mathrm{Cs}$ & 0.02 & 0.227 & 0.700 & $4.4 \times 10^{-12}$ \\
\hline
\end{tabular}

Figure S6. Model Fit of SAXS curves. Model Fits were performed using SASfit software ${ }^{1}$. a) Dotted line shows the model Fit using a cylindrical form factor $(\mathrm{F}(\mathrm{q}))$ and hard sphere structure factor $(\mathrm{S}(\mathrm{q}))$ )). The Form factor contribution is shown in green, the measured data of $\mathrm{MAPbI}_{3}, \mathrm{MAPbI}_{3}$ with $5 \mathrm{vol} \% \mathrm{CsI}$ and $10 \mathrm{vol} \% \mathrm{CsI}$ are presented as solid line (from left to right). A comparison of the structure factor contribution of each sample is displayed in b) as well as the respective parameters in an inset: the hard-sphere interaction radius RHS, and the volume fraction fp. c) provides schematically the chosen form and structure factor based on the lead coordination as shown in Figure 1 (main text). The scheme is accompanied by a table containing further fit parameters, such as the width parameter $\mathrm{s}$, the radius $\mathrm{R}$, the length of the cylinder $\mathrm{L}$ and the variance of the fit $\sigma^{2}$. 


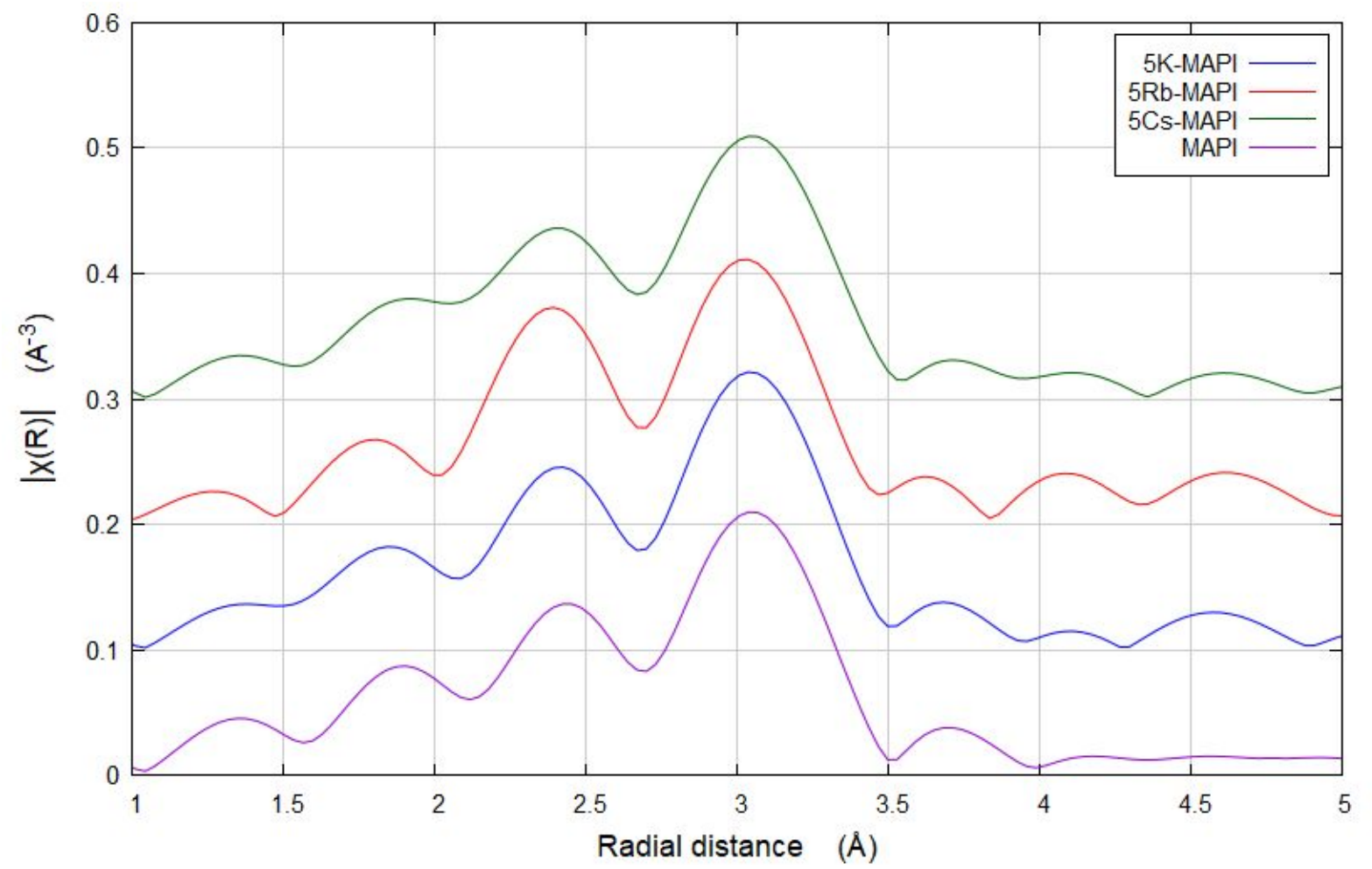

Figure S7. EXAFS of perovskite precursor dispersions. $\mathrm{MAPbI}_{3}$ compared to $\mathrm{MAPbI}_{3}$, including $5 \%$ alkaline salts, $\mathrm{KI}$, RbI, CsI. For clarity, the respective curves are shifted in y-scale.

X-ray absorption spectroscopy measurements performed at $\mathrm{Pb} \mathrm{L}$-edge $(13.035 \mathrm{keV})$ reveal further insights into the lead coordination and interatomic distances of the perovskite precursors. Comparing the $\mathrm{MAPbI}_{3}$ precursor to the $\mathrm{MAPbI}_{3}$ precursor with alkali salt addition provides no significant change in the Fourier Transform of the k-space EXAFS data, presented in Figure S7. This Fourier Transform gives information about the average distance from lead to the most immediate neighboring electron density maximum. Thus, based on this data, we can assume that the additional Iodide provided by the alkali salt does not affect the coordination sphere of lead. 


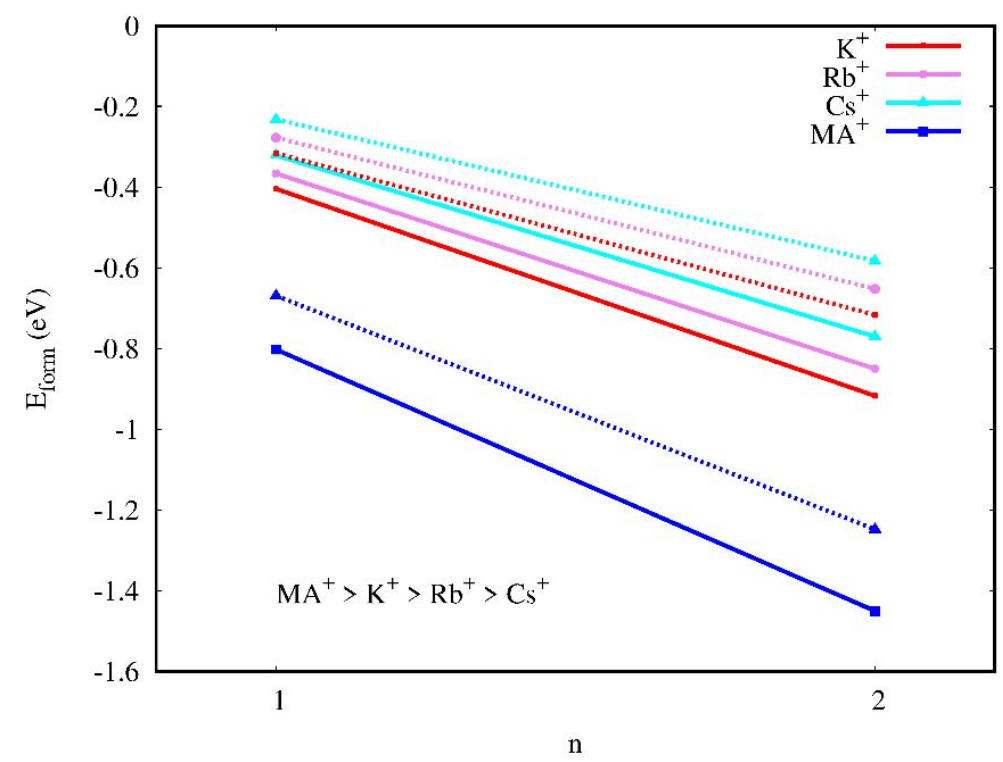

Figure S8. Density functional theory (DFT) calculations of $\mathrm{A}^{+}(\mathrm{solv})_{\mathrm{n}}$ complex formation energy. $\mathrm{E}_{\text {form }}$, as a measure of the solvent-cation interaction, evaluated for $\mathrm{A}=\mathrm{K}, \mathrm{Rb}, \mathrm{Cs}, \mathrm{MA} ; \mathrm{n}=1$ and 2; solv = DMSO (straight line) and DMF (dotted line). Overall $\mathrm{MA}^{+}$shows the highest affinity to be coordinated by solvents and $\mathrm{Cs}^{+}$the lowest.

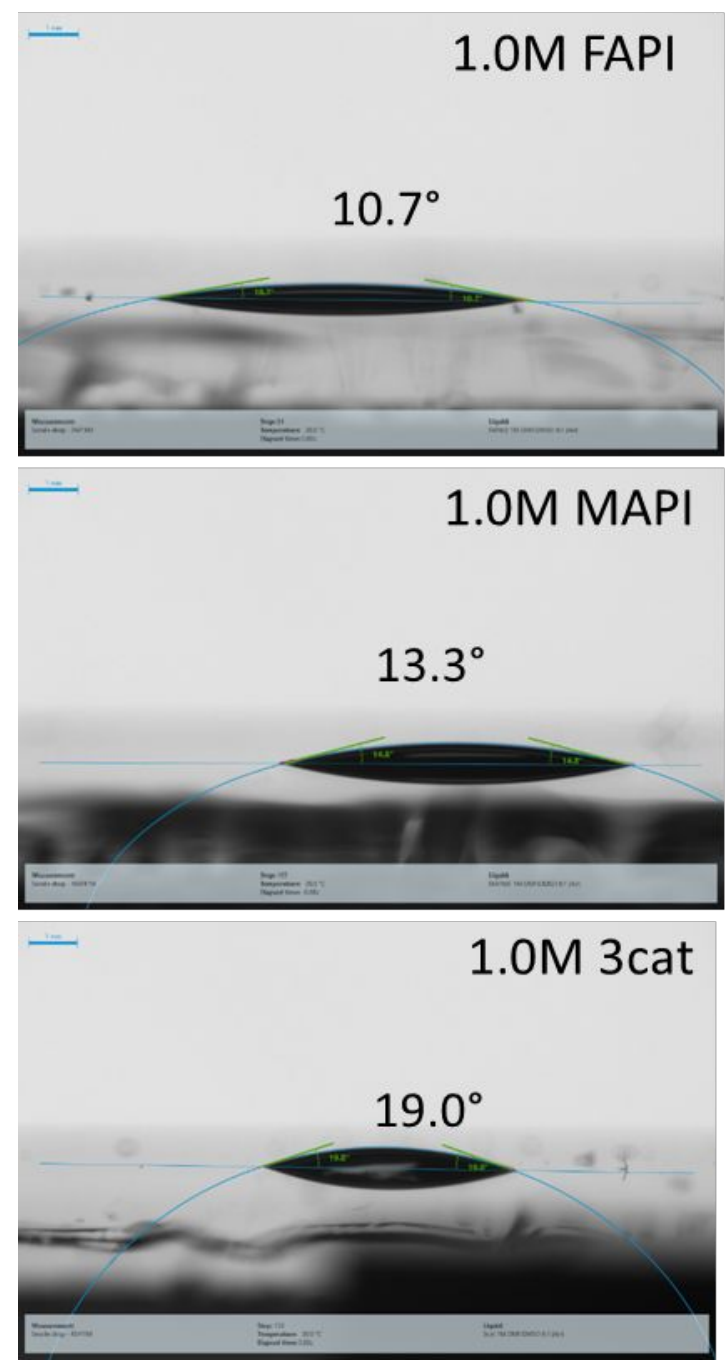

Figure S9. Results of contact angle measurement of $\mathrm{FAPbI}_{3}, \mathrm{MAPbI}_{3}$ and triple cation perovskite solution all in a concentration of $1 \mathrm{~mol} \mathrm{~L}^{-1}$. 

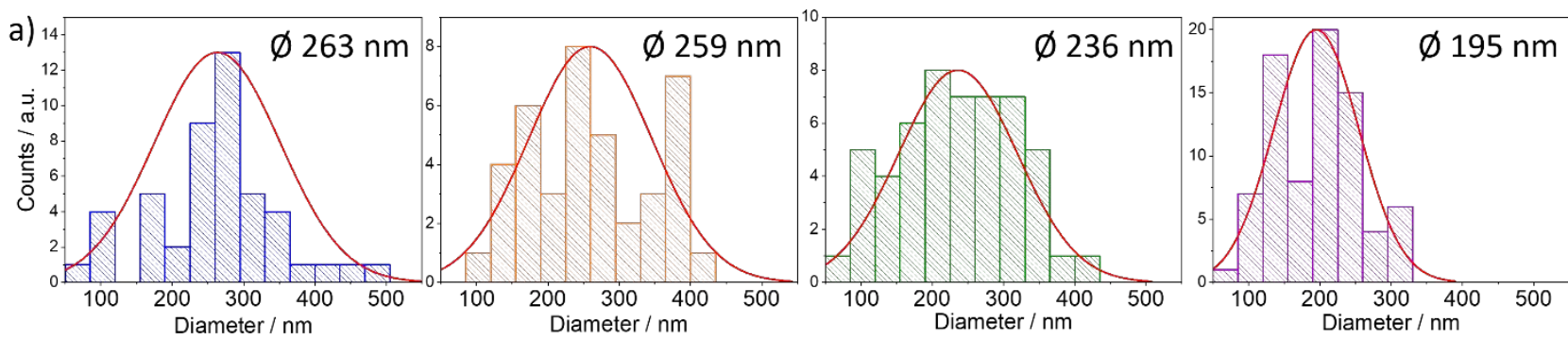

b)

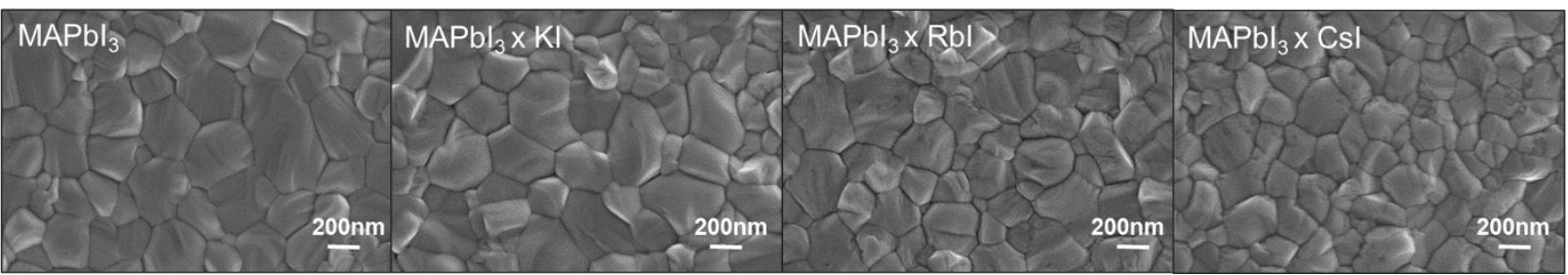

Figure S10. Evolution of the grain size distribution with alkali addition. A comparison of plain $\mathrm{MAPbI}_{3}$ thin film to $\mathrm{MAPbI}_{3}$ with the addition of 5\% KI, RbI, CsI, respectively, where a) shows the overall size distribution presented by the calculated diameter of a grain area (determined using the software Image $\mathrm{J}^{2}$ ), assuming a circular shape of the grains. The mean diameter is given on the top right of each graph. The corresponding SEM images are depicted in b).

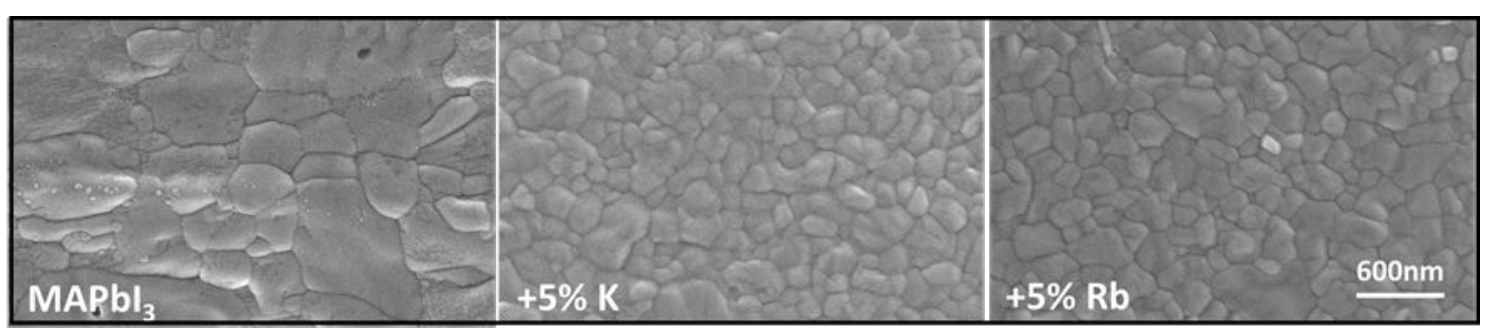

Figure S11. SEM images of $\mathrm{MAbI}_{3}$ thin films compared to $\mathrm{MAPbI}_{3}$ x $5 \% \mathrm{KI}$ and x $5 \% \mathrm{RbI}$ respectively. For thin film preparation the perovskite precursor solution was spread on the cleaned substrate and spin-coated for $5 \mathrm{~s}$ at $1000 \mathrm{rpm}$, no anti-solvent was used.

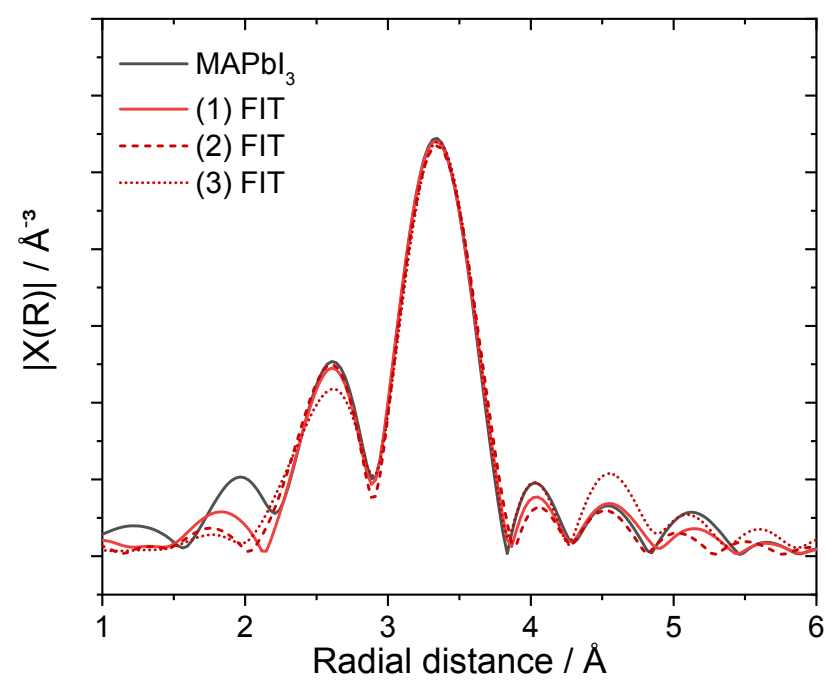

Figure S12. EXAFS data for $\mathrm{MAPbI}_{3}$ perovskite precursor. Different model fits (1)-(2) with the scattering paths provided in Table S1. 
Table S1. EXAFS fit parameters of $\mathrm{MAPbI}_{3}$ considering a different $\mathrm{Pb} 1-\mathrm{O} 1$ contribution.

\begin{tabular}{lllllll}
\hline Sample & R-factor & $\begin{array}{l}\text { Scattering } \\
\text { Path }\end{array}$ & $\mathbf{N}$ & $\mathbf{R}_{\text {model }} / \AA$ & $\mathbf{R}_{\text {fit } /} \AA$ & $\mathbf{R}_{\text {diff }} / \AA$ \\
\hline (1) $\mathrm{MAPb}_{3}$ & 0.007 & $\mathrm{~Pb} 1-11$ & 1 & 3.028 & 2.799 & 0.0525 \\
& & $\mathrm{~Pb} 1-12$ & 2 & 3.177 & 2.948 & 0.0525 \\
& & $\mathrm{~Pb} 1-13$ & 2 & 3.319 & 2.999 & 0.1023 \\
& & $\mathrm{~Pb} 1-\mathrm{O} 1$ & 1 & 4.985 & 4.989 & 0.0000 \\
\hline (2) $\mathrm{MAPbI}_{3}$ & \multirow{2}{*}{0.032} & $\mathrm{~Pb} 1-\mathrm{I1}$ & 1 & 3.028 & 2.860 & -0.1685 \\
& & $\mathrm{~Pb} 1-12$ & 2 & 3.177 & 3.009 & -0.1685 \\
& & $\mathrm{~Pb} 1-13$ & 2 & 3.319 & 3.130 & -0.1892 \\
& & $\mathrm{~Pb} 1-01$ & 1 & 4.985 & 2.485 & -2.5000 \\
\hline (3) $\mathrm{MAPb}_{3}$ & 0.0269 & $\mathrm{~Pb} 1-11$ & 1 & 3.028 & 2.853 & -0.1750 \\
& & $\mathrm{~Pb} 1-12$ & 2 & 3.177 & 3.002 & -0.1750 \\
& & $\mathrm{~Pb} 1-13$ & 2 & 3.319 & 3.115 & -0.2044 \\
& & $\mathrm{~Pb} 1-01$ & 1 & 4.985 & 3.485 & -1.5000 \\
\hline
\end{tabular}




\section{REFERENCES}

(1) Breßler, I.; Kohlbrecher, J.; Thünemann, A. F. SASfit: A Tool for Small-Angle Scattering Data Analysis Using a Library of Analytical Expressions. J. Appl. Crystallogr. 2015, 48, 1587-1598. https://doi.org/10.1107/S1600576715016544.

(2) Schneider, C. A.; Rasband, W. S.; Eliceiri, K. W. NIH Image to ImageJ: 25 Years of Image Analysis. Nat. Methods 2012, 9 (7), 671-675. https://doi.org/10.1038/nmeth.2089. 